\title{
Synthesis of Bioactive Speciosins G and P from Hexagonia speciosa
}

\author{
Guillermo A. Guerrero-Vásquez, Nuria Chinchilla, José M. G. Molinillo, and Francisco A. Macías*
}

Allelopathy Group, Department of Organic Chemistry, School of Sciences, INBIO Institute of Biomolecules, University of Cadiz, C/ República Saharaui, s/n, 11510-Puerto Real, Cádiz, Spain

\section{Supporting Information}

ABSTRACT: The first total synthesis of speciosins P and G, previously isolated from Hexagonia speciosa, is reported. These compounds have been synthesized by Sonogashira coupling from readily available starting materials. Siccayne was also synthesized from the same starting material in two steps along with a number of other derivatives. The compounds were tested in the wheat coleoptile bioassay. The most active compound was the intermediate 18, followed by 29 and 17 . The structural requirements for activity in these compounds are the presence of methoxy groups in the aromatic ring and a formyl or hydroxy group in the side chain.

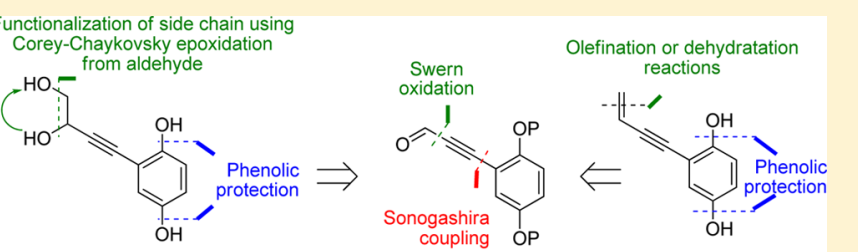

(士)-Speciosin P

Speciosin G
Tn $2009^{1}$ and $2011^{2}$ Jiang and co-workers isolated a series of 1 substituted hydroquinones, named speciosins $\mathrm{A}-\mathrm{T}$, from the Chinese fungus basidiomycete Hexagonia speciosa. Speciosins G (1) and P (2) are structurally similar to the biologically active siccayne (3) (Figure 1), which was isolated from the fungus<smiles>C=CC#Cc1cc(O)ccc1O</smiles>

Speciosin G (1)<smiles>OCC(O)C#Cc1cc(O)ccc1O</smiles>

(士)-Speciosin P (2)

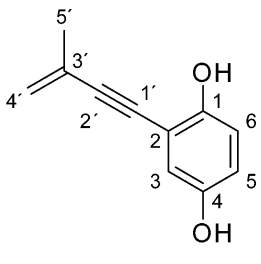

Siccayne (3)
Figure 1. Structures of speciosin G (1), ( \pm )-speciosin P (2), and siccayne (3).

Helminthosporium siccans in 1968 and the marine basidiomycete Halocyphina villosa in 1981..$^{3,4}$ Related compounds such as siccayne, which are present in bacteria, fungi, higher plants, and mollusks, have a wide range of bioactivities.

Compound 3 showed moderate antibiotic activity, inhibited mitochondrial respiration in Saccharomyces cerevisiae, ${ }^{5}$ and showed cytotoxic activity against the human cancer cell lines HeLa and HT29, 5,6 making speciosins G and P potential targets in the development of drugs or pesticides.

A retrosynthetic analysis for compounds $\mathbf{1}$ and $\mathbf{2}$ is shown in Scheme 1 . The key point in this strategy is the attachment of the carbon chain to the aromatic nucleus. The starting materials required to generate the corresponding aryl bromide 6 are readily available and cheap [ $p$-benzoquinone (4), $p$-dimethoxybenzene (5)].

Compound 6 could be linked with propargyl alcohol 7 by Pdcatalyzed Sonogashira coupling to give $\mathbf{8}$, which in turn would provide key aldehyde 9 by oxidation. Compound 9 would allow access to $\mathbf{1}$ and $\mathbf{2}$ by functionalization reactions.
Scheme 1. Retrosynthetic Analysis for Speciosins G (1) and P (2)

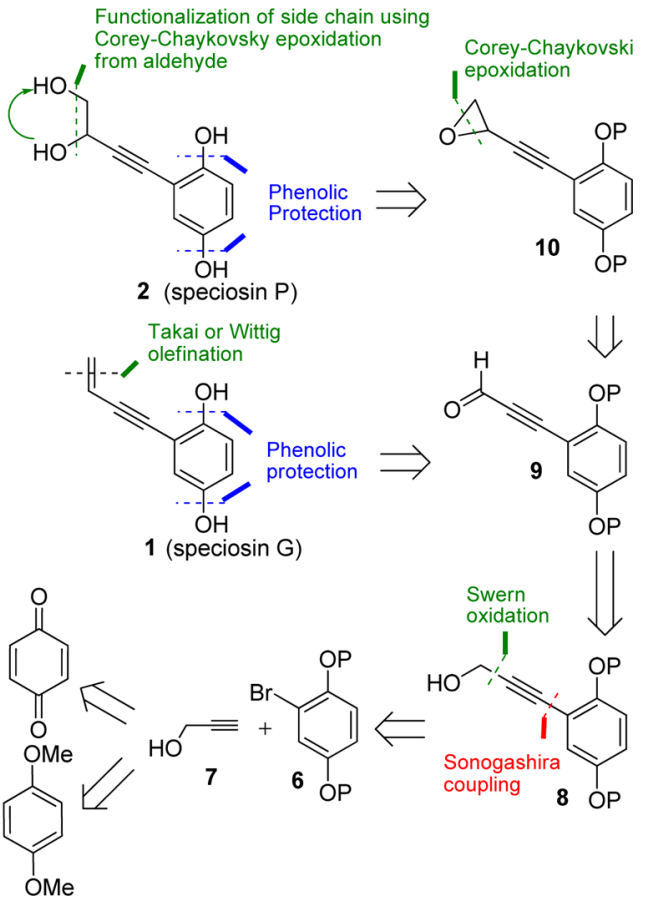

A second aim of this work was the preparation of analogues of these compounds, along with siccayne (3), in order to study their structure-activity relationships.

\section{RESULTS AND DISCUSSION}

Synthesis. Compound $6 a^{7}$ was obtained from $4 .{ }^{8}$ The key reaction is the Sonogashira cross-coupling with propargyl

Received: April 16, 2014

Published: September 2, 2014 
alcohol. $^{9-11}$ This reaction was initially carried out under the conditions reported for the preparation of siccayne (3). In this process $n$-butylamine was used as the solvent and $\mathrm{Pd}\left(\mathrm{PPh}_{3}\right)_{4}$ as the catalyst at $78{ }^{\circ} \mathrm{C}$. The yields obtained using these conditions were about $80 \% .{ }^{8}$ The introduction of $\mathrm{Cu}(\mathrm{I})$ as a cocatalyst led to a decrease in the yield. However, when the reactor was sealed and heated to $100{ }^{\circ} \mathrm{C}$, the yields increased to $96 \%$, i.e., better than previously reported yields (Scheme 2 ). The reaction conditions

Scheme 2. Conditions for the Pd-Cu-Catalyzed CrossCoupling of $\mathrm{sp}^{2}-\mathrm{C}$ Halides with Terminal Acetylenes

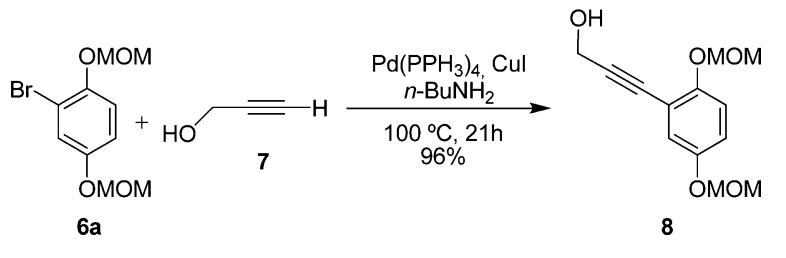

involved the use of equimolecular amounts of aryl bromide $\mathbf{6 a}$ and propargyl alcohol (7) in the presence of the commercially available catalyst tetrakis(triphenylphosphine)palladium $(0)$ (3-5 mol \%) and $n$-butylamine as solvent.

The feasibility of the oxidation of $8^{12}$ to aldehyde 9 was assessed using the Corey-Suggs reagent, pyridinium chlorochromate (PCC), ${ }^{13-15}$ Dess-Martin periodinane (DMP), ${ }^{16}$ and Swern oxidation (Scheme 3). ${ }^{17}$ The latter conditions were selected and permitted scale-up to $3.1 \mathrm{mmol}$ with $91 \%$ yield.

Scheme 3. Synthesis of the Key Aldehyde 9

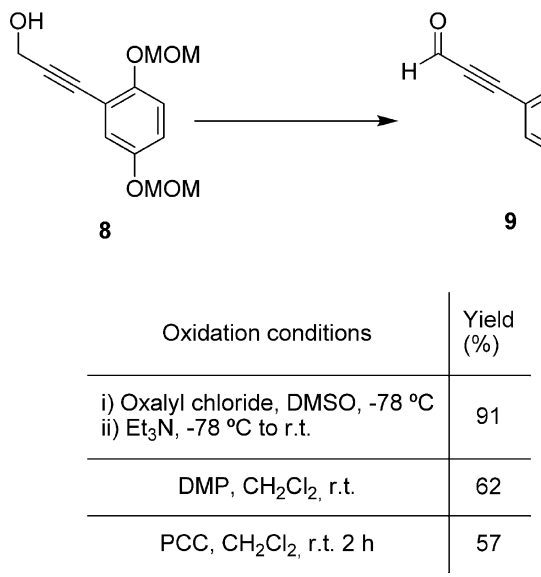

Homologation of the side chain was achieved by the CoreyChaykovsky reaction (Scheme 4). ${ }^{18-20}$ Thus, epoxide 10 was obtained by in situ generation of the dimethylsulfonium methylide $^{12}$ and subsequent addition of a dichloromethane solution of the aldehyde 9. Quantitative acid-mediated hydrolysis of epoxide 10 was performed in acetone $/ \mathrm{H}_{2} \mathrm{SO}_{4}$ to give compound 11. Deprotection of the OMOM groups was carried out in a solution of $\mathrm{HCl}$ in $\mathrm{MeOH}$ to give $( \pm)$-speciosin $\mathrm{P}(2)$ in $90 \%$ yield $\left(72 \%\right.$ overall yield). ${ }^{2}$ Simultaneous hydrolysis and deprotection was not possible. When this reaction was carried out in $\mathrm{MeOH} / \mathrm{H}_{2} \mathrm{SO}_{4}$, the methoxy derivative 12 of $( \pm)$-speciosin P (2) was obtained.

The synthesis of speciosin $\mathrm{G}$ requires olefination of aldehyde 9 (Scheme 5). Wittig reaction of 9 with methyltriphenylphosphonium iodide under standard conditions ${ }^{21}$ provided compound 13 after decarbonylation, as observed previously for other alkynyl
Scheme 4. Synthesis of $( \pm)$-Speciosin P (2) through CoreyChaykovsky Epoxidation

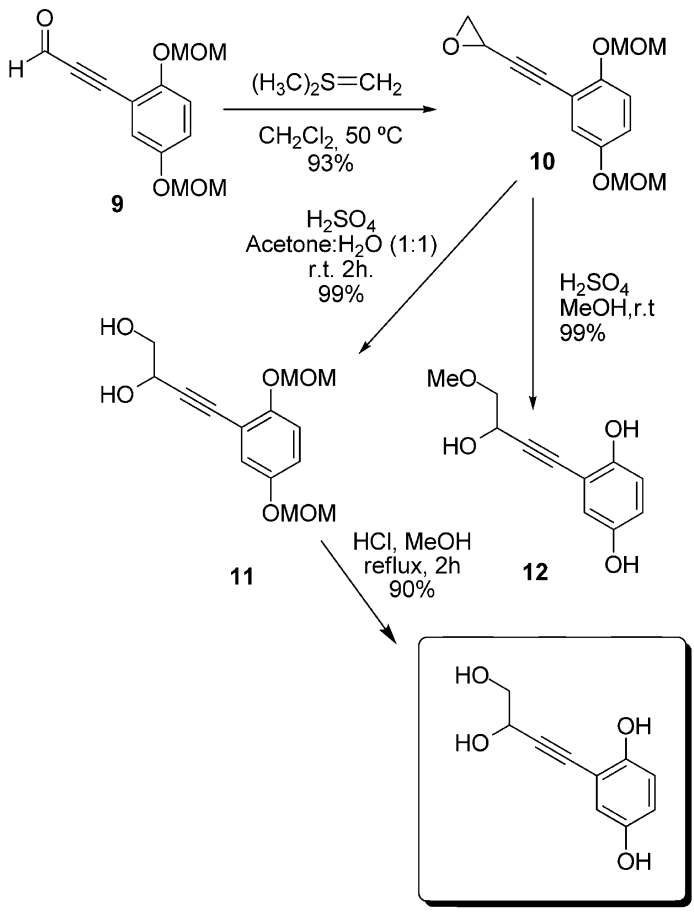

(士)-speciosin P (2)

Scheme 5. Synthesis of Speciosin G (1) through Olefination of the Key Intermediate 9 and Burgess Dehydration of 15
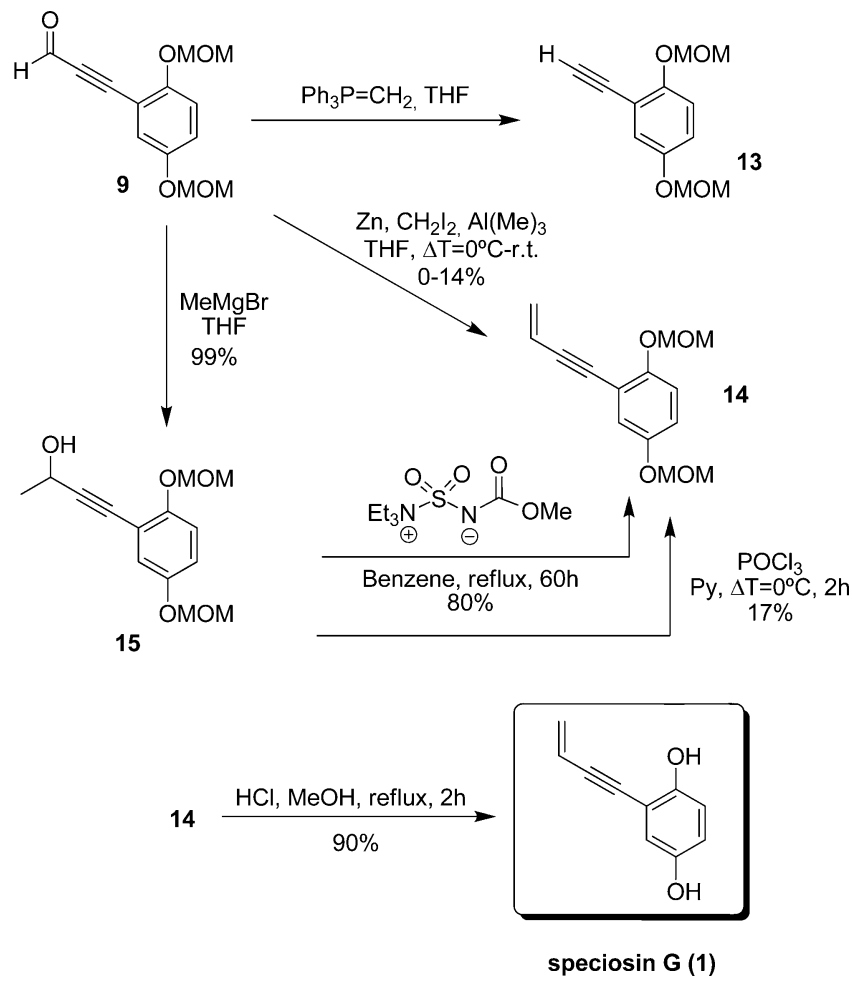

aldehydes. $^{22}$ The modification reported by Takai, in which zinc dust, trimethylaluminum, and $\mathrm{CH}_{2} \mathrm{I}_{2}$ are used, was also employed to synthesize 14, albeit in a yield less than $15 \%$. Alternatively, Grignard coupling ${ }^{23}$ gave a satisfactory yield of 15 , and this was subsequently dehydrated. The commonly used method with 
$\mathrm{POCl}_{3}$ led to poor yields (17-20\%). The use of Burgess' reagent (6 equiv added in three portions over $60 \mathrm{~h}$ ) under reflux and dry conditions gave $\mathbf{1 4}$ in $80 \%$ yield. $^{24}$ Deprotection under acidic conditions yielded speciosin G (1) in $90 \%$ yield (62\% overall yield).

The presence of hydroxy and/or methoxy groups can markedly change the bioactivity of phenolic compounds. ${ }^{25}$ Thus, we also prepared all of the intermediates with methoxy groups in the aromatic ring. Several intermediates of speciosins were also deprotected to give the corresponding hydroxy compounds for subsequent assay. The reactions are summarized in Scheme 6.

\section{Scheme 6. Preparation of the Methoxy and Deprotected} Derivatives

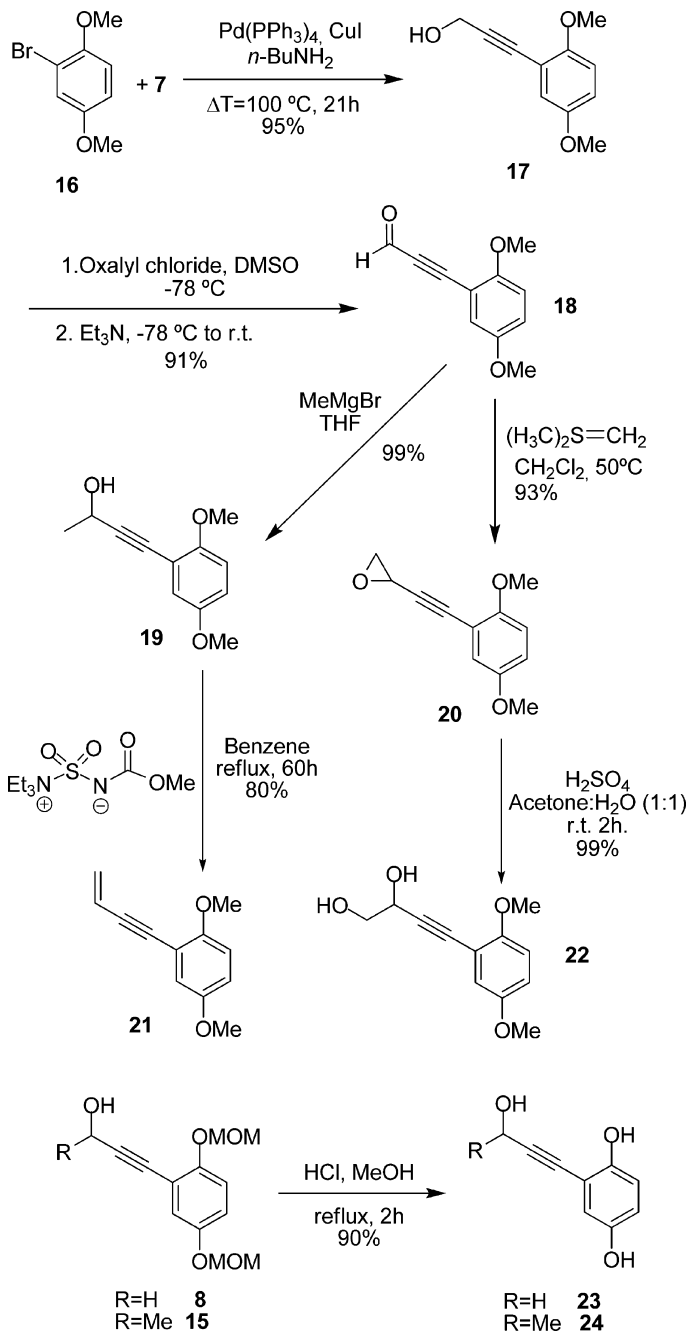

Siccayne (3) and its derivatives were also target compounds. Siccayne (3) was synthesized in $94 \%$ yield by direct Sonogashira reaction with the MOM-protected bromohydroquinone $\mathbf{6 a}$. The initial reaction temperature was $0{ }^{\circ} \mathrm{C}$, and this was increased gradually to $100{ }^{\circ} \mathrm{C}$ due to the volatility of alkyne 25 . Deprotection also gave siccayne (3) in good yield (90\%) under the conditions used for speciosins. Modification of the initial chain of 25 to give diol $26^{26}$ and acetate 27 permitted the synthesis of siccayne derivatives with or without methoxy groups in the aromatic ring (Scheme 7). The acetyl group was removed during the Sonogashira reaction in all cases. It was not possible to obtain
Scheme 7. Preparation of the Racemic Syccaine Derivatives
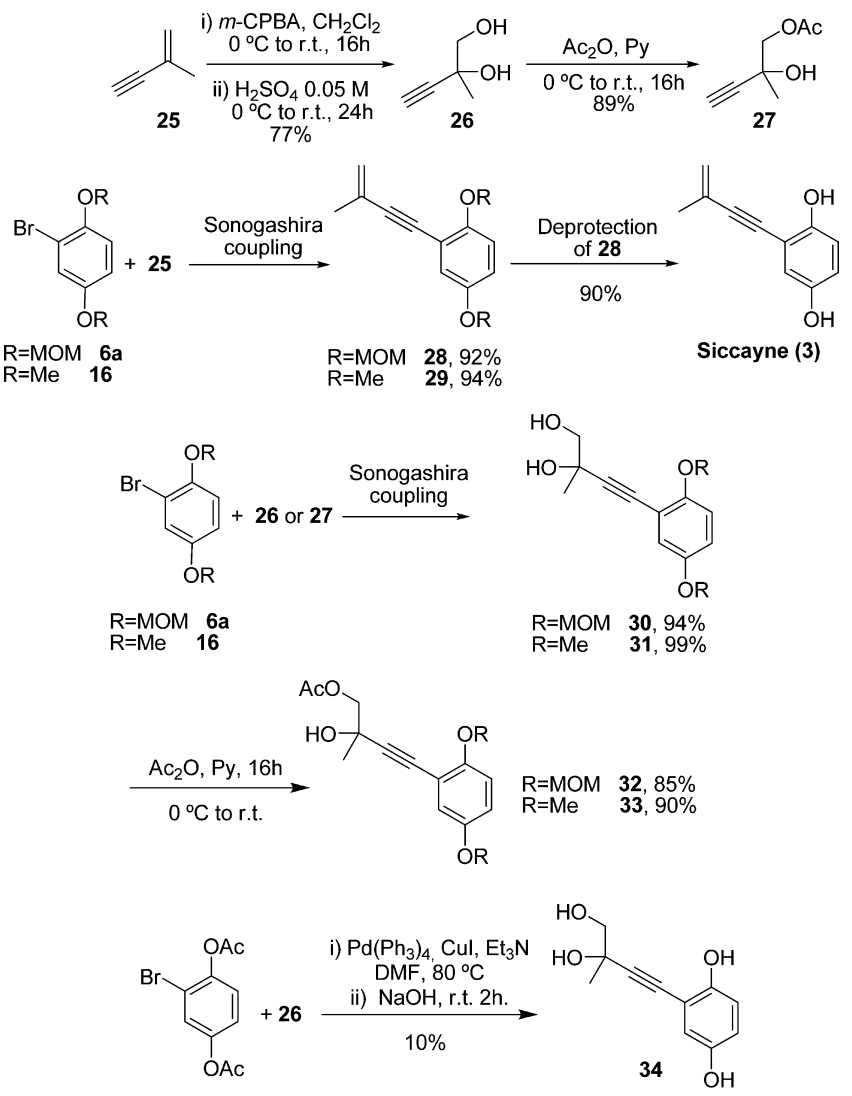

$6 b$

compound 34 by deprotection of 30 . The acetylated derivative of 6 was prepared by Sonogashira coupling of $\mathbf{6 b} .^{7}$

The preparation of speciosin G (1) and ( \pm )-speciosin P (2) was optimized by carrying out a number of Sonogashira reactions under different conditions, which included changes in temperature, the use of $\mathrm{CuI}$ as a cocatalyst, and altering the number of equivalents of reagents. The results are summarized in Table 1. The presence of primary hydroxy groups in the alkynyl chain at $100{ }^{\circ} \mathrm{C}$ was better tolerated when $\mathrm{CuI}$ was not used (entries 3, 6, 16 , and 17). The presence of tertiary hydroxy groups did not have any influence, and better results were obtained on using a cocatalyst (entries 18 and 24). In general, higher yields were obtained by increasing the reaction temperature. It was not necessary to use a temperature above $100{ }^{\circ} \mathrm{C}$, and this led to low overpressure and allowed the accurate control of the reaction.

Speciosins G (1) and P (2) have been synthesized for the first time in overall yields of $72 \%$ and $62 \%$, respectively, from $6 a$ in five steps. Siccayne (3) was also synthesized from the same starting material in two steps with an overall yield of $83 \%$. This procedure was simpler and more efficient than the reported process. ${ }^{8,27}$

Bioactivity. A total of 16 compounds were evaluated by the wheat coleoptile bioassay. This is a rapid test that is sensitive to a wide range of bioactive substances, including plant growth regulators, herbicides, antimicrobials, mycotoxins, and assorted pharmaceuticals. ${ }^{28-30}$ The results are shown in Figure 2, in which negative values signify inhibition, positive values denote activation, and zero represents control.

The most active compound was 18 , followed by 29 and 17 . This trend can be seen more clearly by calculating the $\mathrm{IC}_{50}$ values for the assayed compounds (Table 2), which have values of 323, 670 , and $952 \mu \mathrm{M}$, respectively. 
Table 1. Optimization of the Conditions for the Sonogashira Reaction $^{a}$

\begin{tabular}{|c|c|c|c|c|c|}
\hline entry & substrates & $\begin{array}{c}\mathrm{Pd}\left(\mathrm{PPh}_{3}\right)_{4} \\
(\mathrm{~mol} \mathrm{\%})\end{array}$ & $\begin{array}{c}\mathrm{Cu}(\mathrm{I}) \\
(\mathrm{mol} \%)\end{array}$ & $T\left({ }^{\circ} \mathrm{C}\right)$ & $\begin{array}{c}\text { product (yield, } \\
\%)\end{array}$ \\
\hline 1 & $6+7^{b}$ & 10 & & 78 & $8(82)$ \\
\hline 2 & & 5 & 3 & 100 & $8(59)$ \\
\hline 3 & & 5 & & 100 & $8(96)$ \\
\hline 4 & $16+7^{b}$ & 5 & 3 & 100 & $17(62)$ \\
\hline 5 & & 3 & 3 & 100 & $17(52)$ \\
\hline 6 & & 5 & & 100 & $17(95)$ \\
\hline 7 & $6+25^{c}$ & 3 & 3 & 0 to 100 & $28(87)$ \\
\hline 8 & & 5 & 3 & 0 to 100 & $28(92)$ \\
\hline 9 & $16+25^{c}$ & 3 & 3 & 0 to 100 & $29(72)$ \\
\hline 10 & & 5 & 3 & 0 to 100 & $29(94)$ \\
\hline 11 & & 5 & 5 & 25 & $29(75)$ \\
\hline 12 & $6+26^{d}$ & 5 & 3 & 100 & $30(94)$ \\
\hline 13 & & 5 & & 100 & $30(94)$ \\
\hline 14 & $16+26^{d}$ & 5 & 3 & 100 & $31(94)$ \\
\hline 15 & & 3 & 3 & 100 & $31(12)$ \\
\hline 16 & & 5 & & 100 & 31 (99) \\
\hline 17 & & 3 & & 100 & $31(81)$ \\
\hline 18 & $6+27^{c}$ & 5 & 3 & 100 & $30(80)$ \\
\hline 19 & & 3 & 3 & 100 & $30(31)$ \\
\hline 20 & & 5 & & 100 & $30(65)$ \\
\hline 21 & & 3 & & 100 & $30(62)$ \\
\hline 22 & & 3 & 3 & 78 & $30(44)$ \\
\hline 23 & & 3 & & 78 & $30(41)$ \\
\hline 24 & $16+27^{c}$ & 5 & 3 & 100 & $31(91)$ \\
\hline 25 & & 3 & 3 & 100 & $31(34)$ \\
\hline 26 & & 5 & & 100 & $31(72)$ \\
\hline 27 & & 3 & & 100 & $31(45)$ \\
\hline 28 & & 3 & 3 & 78 & $31(33)$ \\
\hline 29 & & 3 & & 78 & $31(30)$ \\
\hline
\end{tabular}

${ }^{a}$ The reaction time was $21 \mathrm{~h}$ in all cases. The solvent was $n \mathrm{BuNH}_{2}$ except for entries 22, 23, 28, and 29, where TEA, DIPEA, and THF were used. ${ }^{b} 1$ equiv. ${ }^{c} 1.2$ equiv. ${ }^{d} 1.1$ equiv.

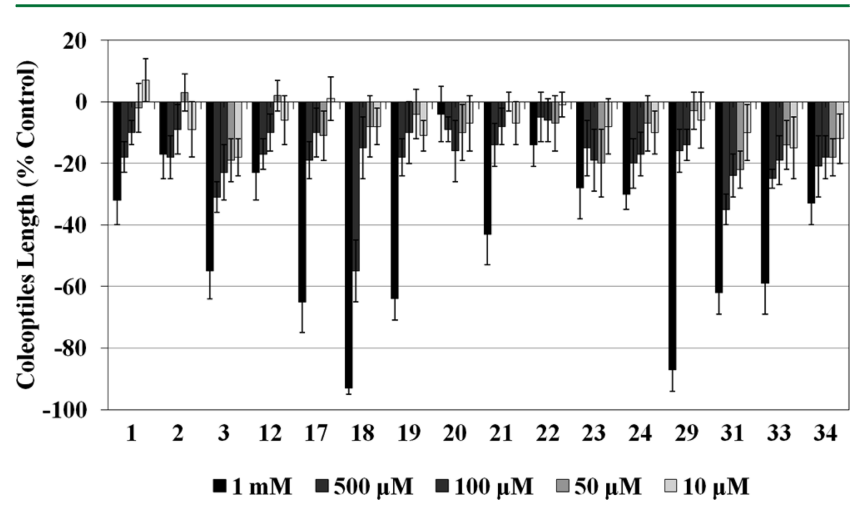

Figure 2. Effect of the compounds tested on etiolated wheat coleoptiles. The bioassays were carried out in triplicate.

Regarding the structural requirements for activity, comparison of pairs $(29,3),(31,34)$, and $(21,1)$ shows that the presence of the methoxy group led to an increase in activity. On the other hand, the compounds with only one hydroxy group in the chain are more active than those that contain two hydroxy groups [cf. $(22,19)$ and $(2,24)]$. Comparison of the siccayne $(3,29,31$, and 34) and speciosin $(1,2,21$, and 22) derivatives revealed that the siccayne derivatives are more active than the speciosins.

In summary, a number of derivatives were prepared and tested in the wheat coleoptile bioassay. The optimum structural
Table 2. $\mathrm{IC}_{50}$ Values Obtained in the Wheat Coleoptile Bioassay

\begin{tabular}{cclccc} 
compound & $\mathrm{IC}_{50}(\mu \mathrm{M})$ & \multicolumn{1}{c}{$R^{2}$} & compound & $\mathrm{IC}_{50}(\mu \mathrm{M})$ & $R^{2}$ \\
$\mathbf{1}$ & 2568 & 0.9867 & $\mathbf{2 1}$ & 2010 & 0.9922 \\
$\mathbf{2}$ & 14440 & 0.9804 & $\mathbf{2 2}$ & 62260 & 0.9958 \\
$\mathbf{3}$ & 1253 & 0.9682 & $\mathbf{2 3}$ & 7890 & 0.9638 \\
$\mathbf{1 2}$ & 5799 & 0.9857 & $\mathbf{2 4}$ & 4354 & 0.9826 \\
$\mathbf{1 7}$ & 952.4 & 0.9753 & $\mathbf{2 9}$ & 670.2 & 0.9174 \\
$\mathbf{1 8}$ & 322.6 & 0.974 & $\mathbf{3 1}$ & 821 & 0.9731 \\
$\mathbf{1 9}$ & 1055 & 0.9703 & 33 & 1160 & 0.977 \\
$\mathbf{2 0}$ & & & 34 & 4434 & 0.9724 \\
\hline
\end{tabular}

requirements for activity in these compounds are the presence of methoxy groups in the aromatic ring and a formyl or hydroxy group in the chain.

\section{EXPERIMENTAL SECTION}

General Experimental Procedures. Commercially available reagents and solvents were analytical grade or were purified by standard procedures prior to use. Compounds were analyzed by $\mathrm{IR},{ }^{1} \mathrm{H} N \mathrm{NR}$, and ${ }^{13} \mathrm{C}$ NMR spectroscopy and by high-resolution ESI mass spectrometry. The data obtained are consistent with the proposed structures. Infrared spectra were recorded on a PerkinElmer FT-IR Spectrum 1000 Mattson 5020 system. HRMS were obtained on a Waters SYNAPT G2 mass spectrometer $(70 \mathrm{eV}) .{ }^{1} \mathrm{H} \mathrm{NMR},{ }^{1} \mathrm{H}-{ }^{1} \mathrm{H}$ gCOSY,${ }^{1} \mathrm{H}-{ }^{13} \mathrm{C}$ gHSQC, and ${ }^{1} \mathrm{H}-{ }^{13} \mathrm{C}$ gHMBC NMR spectra were recorded on Agilent INOVA-400 and INOVA-500 spectrometers using $\mathrm{CDCl}_{3}$ or methanol- $d_{4}$. Chemical shifts $(\delta)$ are reported in parts per million (ppm) relative to either a TMS internal standard or solvent signals. HRMS data were obtained on a Waters SYNAPT G2 mass spectrometer $(70 \mathrm{eV})$. Column chromatography was performed on silica gel (35-75 mesh), and TLC analysis was carried out using aluminum precoated silica gel plates. Synthetic products were purified by preparative HPLC using a Lichrosorb silica 60 semipreparative column (Lichrospher $\mathrm{SiO}_{2}$, Merck, 7 and $10 \mu \mathrm{m}, 150 \times 10 \mathrm{~nm}$ ) and Lichrosorb silica 60 analytical columns (Lichrospher $\mathrm{SiO}_{2}$, Merck, 7 and $10 \mu \mathrm{m}, 250 \times 10 \mathrm{~mm}$ ) in conjunction with a Hitachi Lachrom D-7000 PLC system with a Hitachi L-7490 RI detector and Hitachi L-7420 UV detector.

General Procedure for the Sonogashira Coupling., 8,10,11 Compounds $6 a^{31}$ and $16^{8}$ were synthesized according to literature procedures. Aryl halide $6 \mathbf{a}$ or $\mathbf{1 6}(9.21 \mathrm{mmol})$ in $n$-butylamine $(6.4 \mathrm{~mL})$ was placed in a flame-dried round-bottomed flask under an argon atmosphere. A mixture of terminal alkynes 7, 25, 26, or 27 (9.21 mmol) in $n$-butylamine $(10 \mathrm{~mL})$ and $\mathrm{Pd}\left(\mathrm{Ph}_{3}\right)_{4}(5 \%$ or $3 \%)$ was added, with the optional addition of $\mathrm{CuI}(3 \%)$ where appropriate. The mixture was heated for $21 \mathrm{~h}$ at $98^{\circ} \mathrm{C}$ and poured into $\mathrm{H}_{2} \mathrm{O}(80 \mathrm{~mL})$. The product was extracted with EtOAc $(3 \times 80 \mathrm{~mL})$. The combined organic layers were washed with brine, dried over anhydrous $\mathrm{Na}_{2} \mathrm{SO}_{4}$, and evaporated under reduced pressure. The crude product was purified by silica gel column chromatography (EtOAc/hexanes, 10-50\%).

3-[2,5-Bis(methoxymethoxy)phenyl]prop-2-yn-1-ol ${ }^{12}$ (8). Yield 96\%; colorless oil; IR (KBr) $\nu_{\max } 3310,2230 \mathrm{~cm}^{-1}$; ${ }^{1} \mathrm{H} \mathrm{NMR}\left(\mathrm{CDCl}_{3}\right.$, $400 \mathrm{MHz}) \delta 3.46(3 \mathrm{H}, \mathrm{s}, \mathrm{H}-4 \mathrm{~b}), 3.51(3 \mathrm{H}, \mathrm{s}, \mathrm{H}-1 \mathrm{~b}), 4.51(2 \mathrm{H}, \mathrm{s}, \mathrm{H}-1 \mathrm{a})$, $5.09(2 \mathrm{H}, \mathrm{s}, \mathrm{H}-4 \mathrm{a}), 5.17(2 \mathrm{H}, \mathrm{s}, \mathrm{H}-1 \mathrm{a}), 6.95(1 \mathrm{H}, \mathrm{dd}, J=9$ and $3.0 \mathrm{~Hz}$, $\mathrm{H}-5), 7.03(1 \mathrm{H}, \mathrm{d}, J=9.0 \mathrm{~Hz}, \mathrm{H}-6), 7.10(1 \mathrm{H}, \mathrm{d}, J=3.0 \mathrm{~Hz}, \mathrm{H}-3) ;{ }^{13} \mathrm{C}$ NMR $\left(\mathrm{CDCl}_{3}, 100 \mathrm{MHz}\right) \delta 51.81$ (C-9), $56.05(\mathrm{C}-4 \mathrm{~b}), 56.38(\mathrm{C}-1 \mathrm{~b})$, 81.74 (C-7), 91.56 (C-8), 95.14 (C-4a), 95.88 (C-4b), 114.19 (C-2), 117.13 (C-5), 118.50 (C-3), 121.20 (C-6), 151.95 (C-4), 153.06 (C-1); HRESIMS $m / z 275.0900[\mathrm{M}+\mathrm{Na}]^{+}\left(\right.$calcd for $\left.\mathrm{C}_{13} \mathrm{H}_{16} \mathrm{O}_{5} 275.0896\right)$.

3-(2,5-Dimethoxyphenyl)prop-2-yn-1-ol ${ }^{12}$ (17). Yield 95\%; yellow microcrystalline solid; mp $67-68{ }^{\circ} \mathrm{C}$; IR $(\mathrm{KBr}) \nu_{\max } 2230,3396 \mathrm{~cm}^{-1}$; ${ }^{1} \mathrm{H} \mathrm{NMR}\left(\mathrm{CDCl}_{3}, 400 \mathrm{MHz}\right) \delta 3.73(3 \mathrm{H}, \mathrm{s}, \mathrm{H}-4 \mathrm{a}), 3.82(3 \mathrm{H}, \mathrm{s}, \mathrm{H}-1 \mathrm{a})$, $4.52(2 \mathrm{H}, \mathrm{s}, \mathrm{H}-9), 6.78(1 \mathrm{H}, \mathrm{d}, J=8.3 \mathrm{~Hz}, \mathrm{H}-6), 6.83(1 \mathrm{H}, \mathrm{dd}, J=9.0$ and $3.0 \mathrm{~Hz}, \mathrm{H}-5), 6.94(1 \mathrm{H}, \mathrm{d}, J=3.0 \mathrm{~Hz}, \mathrm{H}-3) ;{ }^{13} \mathrm{C} \mathrm{NMR}\left(\mathrm{CDCl}_{3}, 100\right.$ MHz) $\delta 51.76$ (C-9), 55.84 (C-4a), 56.43 (C-1a), 81.80 (C-7), 91.62 (C-8), 111.99 (C-3), 112.24 (C-2), 115.92 (C-5), 118.43 (C-6), 153.23 
(C-1), 154.49 (C-4); HRESIMS $m / z 215.0694[\mathrm{M}+\mathrm{Na}]^{+}$(calcd for $\mathrm{C}_{11} \mathrm{H}_{12} \mathrm{O}_{3}$ 215.0685).

1,4-Bis(methoxymethoxy)-2-(3-methylbut-3-en-1-yn-1-yl)benzene $^{8}$ (28). Yield 92\%; colorless oil; IR $(\mathrm{KBr}) \nu_{\max } 2194,1493 \mathrm{~cm}^{-1}$; ${ }^{1} \mathrm{H} \mathrm{NMR}\left(\mathrm{CDCl}_{3}, 400 \mathrm{MHz}\right) \delta 1.99(3 \mathrm{H}, \mathrm{s}, 10-\mathrm{H}), 3.46(3 \mathrm{H}, \mathrm{s}, 4 \mathrm{~b}-\mathrm{H}) *$, $3.51(3 \mathrm{H}, \mathrm{s}, 1 \mathrm{~b}-\mathrm{H}) *, 5.09(2 \mathrm{H}, \mathrm{s}, 4 \mathrm{a}-\mathrm{H}), 5.17(2 \mathrm{H}, \mathrm{s}, 1 \mathrm{a}-\mathrm{H}), 6.95(1 \mathrm{H}, \mathrm{d}$, $J=9.0$ and $3.0 \mathrm{~Hz}, 5-\mathrm{H}), 7.03(\mathrm{~d}, J=9.0 \mathrm{~Hz}, 1 \mathrm{H}, 6-\mathrm{H}), 7.10(1 \mathrm{H}, \mathrm{d}, J=$ $3.0 \mathrm{~Hz}, 3-\mathrm{H}) ;{ }^{13} \mathrm{C} \mathrm{NMR}\left(\mathrm{CDCl}_{3}, 100 \mathrm{MHz}\right) \delta 23.40(\mathrm{C}-10), 55.92(\mathrm{C}-$ 4a)*, 56.38 (C-1a)*, 84.44 (C-7), 94.51 (C-8), 95.01 (C-4b)*, 95.81 $(\mathrm{C}-1 \mathrm{~b}) *, 113.30$ (C-2), 116.35 (C-5), 117.48 (C-3), 121.39 (C-6), 121.94 (C-11), 126.85 (C-9), 151.91 (C-4), 152.70 (C-1); HRESIMS $m / z 263.1286[\mathrm{M}+\mathrm{H}]^{+}$(calcd. for $\mathrm{C}_{15} \mathrm{H}_{18} \mathrm{O}_{4} 263.1284$ ).

1,4-Dimethoxy-2-(3-methylbut-3-en-1-yn-1-yl)benzene 32 (29). Yield 94\%; yellow oil; IR (KBr) $\nu_{\max } 1670,2192 \mathrm{~cm}^{-1}$; ${ }^{1} \mathrm{H}$ NMR $\left(\mathrm{CDCl}_{3}, 400 \mathrm{MHz}\right) \delta 2.0(3 \mathrm{H}, \mathrm{s}, \mathrm{H}-11), 3.75(3 \mathrm{H}, \mathrm{s}, \mathrm{H}-4 \mathrm{a}), 3.82(3 \mathrm{H}, \mathrm{s}$, $\mathrm{H}-1 \mathrm{a}), 5.29(1 \mathrm{H}, \mathrm{d}, J=1.6 \mathrm{~Hz}, \mathrm{H}-10 \mathrm{a}), 5.41(1 \mathrm{H}, \mathrm{d}, J=1.6 \mathrm{~Hz}, \mathrm{H}-10 \mathrm{~b})$, $6.78(1 \mathrm{H}, \mathrm{d}, J=9.0 \mathrm{~Hz}, \mathrm{H}-6), 6.81(1 \mathrm{H}, \mathrm{dd}, J=9.0$ and $3.0 \mathrm{~Hz}, \mathrm{H}-5), 7.11$ $(1 \mathrm{H}, \mathrm{d}, J=3.0 \mathrm{~Hz}, \mathrm{H}-3) ;{ }^{13} \mathrm{C} \mathrm{NMR}\left(\mathrm{CDCl}_{3}, 100 \mathrm{MHz}\right) \delta 23.47(\mathrm{C}-11)$, 55.71 (C-4a), 56.42 (C-1a), 84.56 (C-7), 94.54 (C-8), 112.07 (C-3), 112.87 (C-2), 115.60 (C-5), 118.00 (C-3), 121.93 (C-10), 126.87 (C9), $153.15(\mathrm{C}-1), 154.32(\mathrm{C}-4)$; HRESIMS $m / z 203.1073[\mathrm{M}+\mathrm{H}]^{+}$ (calcd for $\mathrm{C}_{13} \mathrm{H}_{14} \mathrm{O}_{2}$ 203.1073).

4-[2,5-Bis(methoxymethoxy)phenyl]-2-methylbut-3-yne-1,2-diol (30). Yield 94\%; colorless oil; IR ( $\mathrm{KBr}) \nu_{\max } 3412,2193 \mathrm{~cm}^{-1}$; ${ }^{1} \mathrm{H}$ NMR $\left(\mathrm{CDCl}_{3}, 400 \mathrm{MHz}\right) \delta 1.53(3 \mathrm{H}, \mathrm{s}, \mathrm{H}-11), 3.45(3 \mathrm{H}, \mathrm{s}, \mathrm{H}-4 \mathrm{~b}), 3.49(3 \mathrm{H}$, s, H-1b), 3.56, 3.73 (each $1 \mathrm{H}, \mathrm{d}, J=11.0 \mathrm{~Hz}, \mathrm{H}-10), 5.08(2 \mathrm{H}, \mathrm{s}, \mathrm{H}-4 \mathrm{a})$, $5.15(2 \mathrm{H}, \mathrm{s}, \mathrm{H}-1 \mathrm{a}), 6.93(1 \mathrm{H}, \mathrm{d}, J=9.0$ and $2.9 \mathrm{~Hz}, \mathrm{H}-5), 6.98(1 \mathrm{H}, \mathrm{d}$, $J=9.0 \mathrm{~Hz}, \mathrm{H}-6), 7.07(1 \mathrm{H}, \mathrm{d}, J=2.9 \mathrm{~Hz}, \mathrm{H}-3) ;{ }^{13} \mathrm{C} \mathrm{NMR}\left(\mathrm{CDCl}_{3}\right.$, $100 \mathrm{MHz}) \delta 24.93$ (C-11), 55.88 (C-1a), 56.32 (C-4a), 69.26 (C-9), 70.98 (C-10), 80.62 (C-7), 94.88 (C-8), 94.95 (C-1b), 95.85 (C-4b), 113.76 (C-2), 117.03 (C-6), 118.45 (C-5), 120.50 (C-3), 151.82 (C-1), 152.88 (C-4); HRESIMS $m / z 279.1235\left[\mathrm{M}-\mathrm{H}_{2} \mathrm{O}+\mathrm{H}\right]^{+}$(calcd for $\mathrm{C}_{15} \mathrm{H}_{20} \mathrm{O}_{6}$ 279.1233).

4-(2,5-Dimethoxyphenyl)-2-methylbut-3-yne-1,2-diol (31). Yield $12 \%$; yellow oil; IR (KBr) $\nu_{\max } 2226,3394 \mathrm{~cm}^{-1} ;{ }^{1} \mathrm{H} \mathrm{NMR}\left(\mathrm{CDCl}_{3}, 400\right.$ $\mathrm{MHz}) \delta 1.54$ (3H, s, H-11), 3.73 (3H, s, H-4a), 3.80 (3H, s, H-1a), 3.55, 3.73 (each $1 \mathrm{H}, \mathrm{d}, J=11.0 \mathrm{~Hz}, \mathrm{H}-10), 6.76(1 \mathrm{H}, \mathrm{d}, J=8.0 \mathrm{~Hz}, \mathrm{H}-6), 6.83$ $(1 \mathrm{H}, \mathrm{dd}, J=9.0$ and $3.0 \mathrm{~Hz}, \mathrm{H}-5), 6.89(1 \mathrm{H}, \mathrm{d}, J=3.0 \mathrm{~Hz}, \mathrm{H}-3) ;{ }^{13} \mathrm{C}$ NMR $\left(\mathrm{CDCl}_{3}, 100 \mathrm{MHz}\right) \delta 24.87$ (C-11), 55.70 (C-1a), 56.34 (C-4a), 69.33 (C-9), 71.03 (C-10), 80.87 (C-7), 94.94 (C-8), 111.83 (C-2), 111.89 (C-6), 115.90 (C-5), 117.75 (C-3), 153.12 (C-1), 154.39 (C-4); HRESIMS $m / z 219.0973\left[\mathrm{M}-\mathrm{H}_{2} \mathrm{O}+\mathrm{H}\right]^{+}$(calcd for $\mathrm{C}_{13} \mathrm{H}_{16} \mathrm{O}_{4}$ 235.0971).

Generation of the Key Aldehyde. ${ }^{17}$ Oxalyl chloride $(272.3 \mu \mathrm{L}$, $3.12 \mathrm{mmol})$ in dry $\mathrm{CH}_{2} \mathrm{Cl}_{2}(9 \mathrm{~mL})$ was added to a stirred solution of DMSO $(332 \mu \mathrm{L}, 4.68 \mathrm{mmol})$ in dry $\mathrm{CH}_{2} \mathrm{Cl}_{2}(1.5 \mathrm{~mL})$ under an argon atmosphere at $-78{ }^{\circ} \mathrm{C}$. The mixture was stirred for $15 \mathrm{~min}$, and the alcohol 8 (393.5 mg, $1.56 \mathrm{mmol})$ or alcohol $17(300 \mathrm{mg}, 1.56 \mathrm{mmol})$ in dry $\mathrm{CH}_{2} \mathrm{Cl}_{2}(12 \mathrm{~mL})$ was added dropwise (Note: Swern oxidation could be scaled-up to $1.56 \mathrm{mmol}$ of starting material). After the starting material had been consumed (nearly $2 \mathrm{~h}$ ), $\mathrm{Et}_{3} \mathrm{~N}(1.88 \mathrm{~mL}, 7.8 \mathrm{mmol}$ ) was added. The reaction mixture was stirred at $-78{ }^{\circ} \mathrm{C}$ for a further $30 \mathrm{~min}$ and was allowed to warm to $\mathrm{rt}$ and quenched with saturated $\mathrm{NH}_{4} \mathrm{Cl}$ and $\mathrm{H}_{2} \mathrm{O}$, and the mixture was stirred for $30 \mathrm{~min}$. The organic phase was decanted off, and the aqueous layer was extracted with $\mathrm{CH}_{2} \mathrm{Cl}_{2}(3 \times 30 \mathrm{~mL})$. The combined organic layers were washed with brine, dried over anhydrous $\mathrm{Na}_{2} \mathrm{SO}_{4}$, and evaporated under reduced pressure.

3-[2,5-Bis(methoxymethoxy)phenyl]prop-2-ynal (9). Yield 91\%; colorless oil; IR $(\mathrm{KBr}) \nu_{\max } 1660,2194 \mathrm{~cm}^{-1} ;{ }^{1} \mathrm{H}$ NMR $\left(\mathrm{CDCl}_{3}\right.$, $400 \mathrm{MHz}) \delta 3.46(3 \mathrm{H}, \mathrm{s}, \mathrm{H}-4 \mathrm{~b}), 3.51(3 \mathrm{H}, \mathrm{s}, \mathrm{H}-1 \mathrm{~b}), 5.10(2 \mathrm{H}, \mathrm{s}, \mathrm{H}-4 \mathrm{a})$, $5.21(2 \mathrm{H}, \mathrm{s}, \mathrm{H}-1 \mathrm{a}), 7.09(1 \mathrm{H}, \mathrm{dd}, J=9.2$ and $1.2 \mathrm{~Hz}, \mathrm{H}-6), 7.12(1 \mathrm{H}, \mathrm{dd}$, $J=9.1$ and $2.2 \mathrm{~Hz}, \mathrm{H}-5), 7.22(1 \mathrm{H}, \mathrm{dd}, J=2.2$ and $1.3 \mathrm{~Hz}, \mathrm{H}-3), 9.44$ $(1 \mathrm{H}, \mathrm{s}, \mathrm{H}-9) ;{ }^{13} \mathrm{C} \mathrm{NMR}\left(\mathrm{CDCl}_{3}, 100 \mathrm{MHz}\right) \delta 56.18(\mathrm{C}-4 \mathrm{~b}), 56.54(\mathrm{C}-$ 1b), 92.05 (C-8), 92.27 (C-7), 95.22 (C-4a), 95.58 (C-1a), 110.70 (C2), 116.72 (C-6), 122.0 (C-5), 122.09 (C-3), 151.85 (C-4), 154.88 (C1), 176.92 (C-9); HRESIMS $m / z 273.0741[\mathrm{M}+\mathrm{Na}]^{+}$(calcd for $\mathrm{C}_{13} \mathrm{H}_{14} \mathrm{O}_{5}$ 273.0739).

3-(2,5-Dimethoxyphenyl)propiolaldehyde (18). Yield 91\%; colorless oil; IR $(\mathrm{KBr}) \nu_{\max } 1656,2186 \mathrm{~cm}^{-1}$; ${ }^{1} \mathrm{H} \mathrm{NMR}\left(\mathrm{CDCl}_{3}, 400 \mathrm{MHz}\right) \delta$
3.77 (3H, s, H-4a), 3.88 (3H, s, H-1a), $6.86(1 \mathrm{H}, \mathrm{d}, J=9.0 \mathrm{~Hz}, \mathrm{H}-6), 7.01$ $(1 \mathrm{H}, \mathrm{dd}, J=9.0$ and $3.1 \mathrm{~Hz}, \mathrm{H}-5), 7.05(1 \mathrm{H}, \mathrm{d}, J=3.0 \mathrm{~Hz}, \mathrm{H}-3), 9.45$ $(1 \mathrm{H}, \mathrm{s}, \mathrm{H}-9) ;{ }^{13} \mathrm{C}$ NMR $\left(\mathrm{CDCl}_{3}, 100 \mathrm{MHz}\right) \delta 56.02$ (C-4a), 56.48 (C-1a), 92.37 (C-8), 92.56 (C-7), 108.94 (C-2), 112.36 (C-6), 119.05 (C-5), 119.97 (C-3), 153.29 (C-1), 156.49 (C-4), 176.92 (C-9); HRESIMS $m / z 213.0529[\mathrm{M}+\mathrm{Na}]^{+}$(calcd. for $\left.\mathrm{C}_{11} \mathrm{H}_{10} \mathrm{O}_{3} 213.0528\right)$.

General Procedure for Acetylation. Acetylations were carried out using the standard methodology, which involved dissolving the compound in dry pyridine $(5 \mathrm{~mL})$ and adding an excess of $\mathrm{Ac}_{2} \mathrm{O}$ at $0{ }^{\circ} \mathrm{C}$. The reaction was complete after $16 \mathrm{~h}$, warmed from $0{ }^{\circ} \mathrm{C}$ to room temperature, and quenched by adding distilled $\mathrm{H}_{2} \mathrm{O}$. The product was extracted with EtOAc $(3 \times 25 \mathrm{~mL})$, and the combined organic layers were washed several times with saturated aqueous $\mathrm{CuSO}_{4}$ until the pyridine had been removed. The organic layer was then dried over anhydrous $\mathrm{NaSO}_{4}$, filtered, and concentrated under vacuum.

2-Hydroxy-2-methylbut-3-yn-1-yl acetate ${ }^{33}$ (27). Yield 89\%; colorless oil; IR (KBr) $\nu_{\max } 3278,2116,1737 \mathrm{~cm}^{-1} ;{ }^{1} \mathrm{H}$ NMR $\left(\mathrm{CDCl}_{3}\right.$, $400 \mathrm{MHz}) \delta 1.48(3 \mathrm{H}, \mathrm{s}, \mathrm{H}-4), 2.11(3 \mathrm{H}, \mathrm{s}, \mathrm{H}-7), 2.45$ (1H, s, H-1), 4.10 $(2 \mathrm{H}, \mathrm{dd}, J=7.4$ and $11.1 \mathrm{~Hz}, \mathrm{H}-5) ;{ }^{13} \mathrm{C} \mathrm{NMR}\left(\mathrm{CDCl}_{3}, 100 \mathrm{MHz}\right) \delta$ 20.91 (C-7), 25.98 (C-4), 66.64 (C-1), 70.82 (C-3), 72.53 (C-5), 84.83 (C-2), $171.04(\mathrm{C}-6)$; HRESIMS $\mathrm{m} / z$ 157.0856 $[\mathrm{M}+\mathrm{H}]^{+}$(calcd for $\mathrm{C}_{8} \mathrm{H}_{12} \mathrm{O}_{3}$ 157.0865).

4-[2,5-Bis(methoxymethoxy)phenyl]-2-hydroxy-2-methylbut-3yn-1-yl acetate (32). Yield $85 \%$; colorless oil; IR (KBr) $\nu_{\max } 3278,2116$, $1737 \mathrm{~cm}^{-1}$; ${ }^{1} \mathrm{H}$ NMR $\left(\mathrm{CDCl}_{3}, 400 \mathrm{MHz}\right) \delta 1.57$ (3H, s, H-11), 2.11 (3H, s, H-13), 3.43 (3H, s, H-4b), 3.48 (3H, s, H-1b), 4.10, 4.31 (each $1 \mathrm{H}, \mathrm{d}, J=11.1 \mathrm{~Hz}, \mathrm{H}-10), 5.07$ (3H, s, H-4a), 5.13 (3H, s, H-1a), 6.92 $(1 \mathrm{H}, \mathrm{dd}, J=9.0$ and $2.9 \mathrm{~Hz}, \mathrm{H}-5), 6.98(1 \mathrm{H}, \mathrm{d}, J=9.0 \mathrm{~Hz}, \mathrm{H}-6), 7.05$ $(1 \mathrm{H}, \mathrm{d}, J=2.9 \mathrm{~Hz}, \mathrm{H}-3) ;{ }^{13} \mathrm{C} \mathrm{NMR}\left(\mathrm{CDCl}_{3}, 100 \mathrm{MHz}\right) \delta 20.78(\mathrm{C}-13)$, 25.94 (C-11), 55.85 (C-1a), 56.15 (C-4a), 67.21 (C-9), 70.86 (C-10), 80.34 (C-7), 93.74 (C-8), 94.89 (C-4b), 95.74 (C-4b), 113.81 (C-2), 117.32 (C-5), 118.45 (C-3), 120.84 (C-6), 151.79 (C-4), 152.88 (C-1), 170.77 (C-12); HRESIMS $m / z 321.1342\left[\mathrm{M}-\mathrm{H}_{2} \mathrm{O}+\mathrm{H}\right]^{+}$(calcd for $\mathrm{C}_{17} \mathrm{H}_{22} \mathrm{O}_{7}$ 321.1366).

4-(2,5-Dimethoxyphenyl)-2-hydroxy-2-methylbut-3-yn-1-yl acetate (33). Yield 90\%; yellow oil; IR $(\mathrm{KBr}) \nu_{\max } 3445,2228,1747$ $\mathrm{cm}^{-1}$; ${ }^{1} \mathrm{H} \mathrm{NMR}\left(\mathrm{CDCl}_{3}, 400 \mathrm{MHz}\right) \delta 1.60(3 \mathrm{H}, \mathrm{s}, \mathrm{H}-11), 2.14(3 \mathrm{H}, \mathrm{s}, \mathrm{H}-$ 13), 3.74 (3H, s, H-4a), 3.80 (3H, s, H-1a), 4.11, 4.37 (each $1 \mathrm{H}, \mathrm{d}, J=$ $11.1 \mathrm{~Hz}, \mathrm{H}-10), 6.78(1 \mathrm{H}, \mathrm{d}, J=9.0 \mathrm{~Hz}, \mathrm{H}-6), 6.83(1 \mathrm{H}, \mathrm{dd}, J=9.0$ and $3.1 \mathrm{~Hz}, \mathrm{H}-3), 6.94(1 \mathrm{H}, \mathrm{d}, J=3.6 \mathrm{~Hz}, \mathrm{H}-5) ;{ }^{13} \mathrm{C} \mathrm{NMR}\left(\mathrm{CDCl}_{3}, 100\right.$ MHz) $\delta 20.85$ (C-13), 25.94 (C-11), 55.77 (C-4a), 56.38 (C-1a), 67.38 (C-9), 70.95 (C-10), 80.63 (C-7), 93.62 (C-8), 111.9 (C-3), 112.18 (C-2), 116.04 (C-5), 118.24 (C-6), 153.11 (C-1), 154.59 (C-4), 170.83 (C-12); HRESIMS $m / z 261.1138\left[\mathrm{M}-\mathrm{H}_{2} \mathrm{O}+\mathrm{H}\right]^{+}\left(\right.$calcd for $\mathrm{C}_{15} \mathrm{H}_{18} \mathrm{O}_{5}$ 261.1154).

General Procedure for the Corey-Chaykovsky Epoxidation. ${ }^{20}$ Potassium hydride $(1.8 \mathrm{mmol})$ as a $30 \%$ mineral oil dispersion was placed in a $25 \mathrm{~mL}$ two-necked, round-bottomed flask and washed four times with $10 \mathrm{~mL}$ portions of hexanes by swirling, allowing the hydride to settle, and decanting, in order to remove the mineral oil. In a separate $25 \mathrm{~mL}$ two-necked round-bottomed flask, dry $\mathrm{CH}_{2} \mathrm{Cl}_{2}(1 \mathrm{~mL})$ was added to trimethylsulfonium methyl sulfate ${ }^{20}(1.8 \mathrm{mmol})$ under an argon atmosphere. This solution was added to the first vessel, and the mixture was stirred at $\mathrm{rt}$ for $1 \mathrm{~h}$ to obtain dimethylsulfonium methylide. A solution of aldehyde $9(114 \mathrm{mg}, 0.6 \mathrm{mmol})$ or $18(150 \mathrm{mg}, 0.6 \mathrm{mmol})$ in dry $\mathrm{CH}_{2} \mathrm{Cl}_{2}(3.5 \mathrm{~mL})$ was added, and the reaction mixture was heated under reflux at $60^{\circ} \mathrm{C}$ for $6 \mathrm{~h}$ under argon to generate the epoxides. Water $(10 \mathrm{~mL})$ was added at $\mathrm{rt}$, and the solution was stirred for $30 \mathrm{~min}$. The organic phase was decanted off, and the aqueous phase was extracted with $\mathrm{CH}_{2} \mathrm{Cl}_{2}(3 \times 10 \mathrm{~mL})$. The combined organic phases were washed with brine and dried over anhydrous $\mathrm{Na}_{2} \mathrm{SO}_{4}$. Evaporation of the solvent gave a residue, which was purified on Sephadex LH-20 eluting with 95\% $\mathrm{CH}_{2} \mathrm{Cl}_{2}$ /hexanes.

2-\{[2,5-Bis(methoxymethoxy)phenyl]ethynyl\}oxirane (10). Yield 85\%; IR (KBr) $\nu_{\max } 2231 \mathrm{~cm}^{-1}$; ${ }^{1} \mathrm{H}$ NMR $\left(\mathrm{CDCl}_{3}, 400 \mathrm{MHz}\right) \delta 3.01$ (2H, m, H-10), 3.47 (3H, s, H-4b), $3.51(3 \mathrm{H}, \mathrm{s}, \mathrm{H}-1 \mathrm{~b}), 3.57(1 \mathrm{H}, \mathrm{t}, J=$ $3.4 \mathrm{~Hz}, \mathrm{H}-9), 5.01(2 \mathrm{H}, \mathrm{s}, \mathrm{H}-4 \mathrm{a}), 5.13(2 \mathrm{H}, \mathrm{s}, \mathrm{H}-1 \mathrm{a}), 6.93$ (1H, dd, $J=9.0$ and $2.9 \mathrm{~Hz}, \mathrm{H}-6), 6.99(1 \mathrm{H}, \mathrm{dd}, J=9.0$ and $3 \mathrm{~Hz}, \mathrm{H}-5), 7.08(1 \mathrm{H}, \mathrm{dd}, J=$ 2.9 and $2.9 \mathrm{~Hz}, \mathrm{H}-3) ;{ }^{13} \mathrm{C}$ NMR $\left(\mathrm{CDCl}_{3}, 100 \mathrm{MHz}\right) \delta 40.19$ (C-9), 48.97 (C-10), 55.76 (C-4b), 56.07 (C-1b), 79.41 (C-7), 89.55 (C-8), 
94.86 (C-4a), 95.57 (C-1a), 113.30 (C-2), 116.84 (C-6), 118.66 (C-5), 121.00 (C-3), 151.65 (C-1), 153.24 (C-4); HRESIMS $m / z 287.0905$ $[\mathrm{M}+\mathrm{Na}]^{+}$(calcd for $\left.\mathrm{C}_{14} \mathrm{H}_{16} \mathrm{O}_{5} 287.0895\right)$.

2-[(2,5-Dimethoxyphenyl)ethynyl]oxirane (20). Yield 93\%; colorless oil; IR (KBr) $\nu_{\max } 2192 \mathrm{~cm}^{-1}$; ${ }^{1} \mathrm{H}$ NMR $\left(\mathrm{CDCl}_{3}, 400 \mathrm{MHz}\right) \delta 3.03$ $(2 \mathrm{H}, \mathrm{m}, \mathrm{H}-10), 3.64(1 \mathrm{H}, \mathrm{m}, \mathrm{H}-9), 3.75(3 \mathrm{H}, \mathrm{s}, \mathrm{H}-4 \mathrm{a}), 3.84(3 \mathrm{H}, \mathrm{s}, \mathrm{H}-$ 1a), $6.80(1 \mathrm{H}, \mathrm{d}, J=9.1 \mathrm{~Hz}, \mathrm{H}-6), 6.86(1 \mathrm{H}, \mathrm{dd}, J=9.0$ and $3.1 \mathrm{~Hz}, \mathrm{H}-5)$, $6.95(1 \mathrm{H}, \mathrm{d}, J=3.1 \mathrm{~Hz}, \mathrm{H}-3) ;{ }^{13} \mathrm{C} \mathrm{NMR}\left(\mathrm{CDCl}_{3}, 100 \mathrm{MHz}\right) \delta 40.60$ (C-9), 49.38 (C-10), 55.96 (C-4a), 56.54 (C-1a), 79.88 (C-7), 89.83 (C-8), 111.67 (C-2), 112.15 (C-6), 116.57 (C-5), 118.54 (C-3), 153.27 (C-4), $155.08(\mathrm{C}-1)$; HRESIMS $\mathrm{m} / \mathrm{z} 205.0865[\mathrm{M}+\mathrm{H}]^{+}$(calcd for $\mathrm{C}_{12} \mathrm{H}_{12} \mathrm{O}_{3}$ 205.0865).

Epoxide Hydrolysis. 4-[2,5-Bis(methoxymethoxy)phenyl]but-3yne-1,2-diol (11). Compound 10 ( $42 \mathrm{mg}, 0.16 \mathrm{mmol}$ ) in $\mathrm{H}_{2} \mathrm{O}$ /acetone $(1: 1)(5 \mathrm{~mL})$ was placed in a flame-dried round-bottomed flask under an argon atmosphere. $\mathrm{H}_{2} \mathrm{SO}_{4}(80 \mu \mathrm{L})$ was added dropwise to the solution. The reaction mixture was stirred at $\mathrm{rt}$ for $90 \mathrm{~min}$ under argon, and the mixture quenched with saturated $\mathrm{NH}_{4} \mathrm{Cl}(5 \mathrm{~mL})$ and stirred for a further $30 \mathrm{~min}$. The organic layer was separated. The aqueous layer was extracted with EtOAc $(4 \times 10 \mathrm{~mL})$. The combined organic layers were washed with brine, dried over anhydrous $\mathrm{Na}_{2} \mathrm{SO}_{4}$, and evaporated under reduced pressure. The crude material was purified by flash silica gel column chromatography, eluting with $40 \% \mathrm{CHCl}_{3}$ /acetone to give compound 11 ( $40.4 \mathrm{mg}, 90 \%$ yield) as a gray, amorphous solid: IR $(\mathrm{KBr}) \nu_{\max } 3336,2197 \mathrm{~cm}^{-1} ;{ }^{1} \mathrm{H}$ NMR $\left(\mathrm{CD}_{3} \mathrm{OD}, 400 \mathrm{MHz}\right) \delta 3.42(3 \mathrm{H}$, s, H-4b), 3.48 (3H, s, H-1b), $3.64(1 \mathrm{H}, \mathrm{dd}, J=7.2$ and $11.2 \mathrm{~Hz}, \mathrm{H}-10 \mathrm{a})$, $3.70(1 \mathrm{H}, \mathrm{dd}, J=4.7$ and $10.1 \mathrm{~Hz}, \mathrm{H}-10 \mathrm{~b}), 4.55(1 \mathrm{H}, \mathrm{d}, J=4.7$ and 7.2 Hz, H-9), 5.09 (2H, s, H-4a), 5.15 (2H, s, H-4b), 6.80 (1H, dd, $J=3.0$ and $9.1 \mathrm{~Hz}, \mathrm{H}-5), 6.86(1 \mathrm{H}, \mathrm{d}, J=9.1 \mathrm{~Hz}, \mathrm{H}-6), 6.94(1 \mathrm{H}, \mathrm{d}, J=3.0 \mathrm{~Hz}$, $\mathrm{H}-3) ;{ }^{13} \mathrm{C}$ NMR (CD $\left.\mathrm{OD}, 100 \mathrm{MHz}\right) \delta 56.12$ (C-4a), 56.55 (C-1a), 64.78 (C-9), 67.38 (C-10), 81.98 (C-7), 93.14 (C-8), 96.09 (C-1a), 96.97 (C-4a), 115.62 (C-2), 118.49 (C-6), 119.30 (C-5), 121.93 (C-3), 153.33 (C-1), 156.24 (C-4); HRESIMS $m / z 282.1053[\mathrm{M}+\mathrm{Na}+\mathrm{H}]^{+}$ (calcd for $\mathrm{C}_{12} \mathrm{H}_{18} \mathrm{O}_{6} 282.1080$ ).

4-(2,5-Dimethoxyphenyl)but-3-yne-1,2-diol (22). Compound 20 $(20 \mathrm{mg}, 0.1 \mathrm{mmol})$ in dry $\mathrm{CH}_{2} \mathrm{Cl}_{2}(2 \mathrm{~mL})$ was placed in a flame-dried round-bottomed flask under an argon atmosphere, and $\mathrm{H}_{2} \mathrm{SO}_{4}(1 \mathrm{M}$, $8 \mathrm{~mL}$ ) was added to the solution. The reaction mixture was heated under reflux at $50{ }^{\circ} \mathrm{C}$ for $24 \mathrm{~h}$ under argon. The mixture was cooled to rt and stirred for $16 \mathrm{~h}$, the mixture was cooled to $0{ }^{\circ} \mathrm{C}, \mathrm{NaHCO}_{3}$ was added to give $\mathrm{pH}=7$, and the solution was stirred for $30 \mathrm{~min}$. The organic phase was decanted off, and the aqueous layer was extracted with EtOAc $(3 \times 10 \mathrm{~mL})$. The combined organic layers were washed with brine, dried over anhydrous $\mathrm{Na}_{2} \mathrm{SO}_{4}$, and evaporated under reduced pressure. The crude material was purified by silica gel column chromatography, eluting with $60 \% \mathrm{EtOAc} /$ hexanes, to give compound $22(18.9 \mathrm{mg}, 87 \%$ yield) as a gray, amorphous solid: $\mathrm{IR}(\mathrm{KBr}) \nu_{\max } 3346,2187 \mathrm{~cm}^{-1} ;{ }^{1} \mathrm{H}$ NMR $\left(\mathrm{CDCl}_{3}, 400 \mathrm{MHz}\right) \delta 3.75(3 \mathrm{H}, \mathrm{s}, \mathrm{H}-4 \mathrm{a}), 3.81(2 \mathrm{H}, \mathrm{s}, \mathrm{H}-10), 3.82$ (3H, s, H-1a), 4.70 (1H, m, H-9), $6.79(1 \mathrm{H}, \mathrm{d}, J=9.0 \mathrm{~Hz}, \mathrm{H}-6), 6.85$ $(1 \mathrm{H}, \mathrm{dd}, J=9.0$ and $3.0 \mathrm{~Hz}, \mathrm{H}-5), 6.94(1 \mathrm{H}, \mathrm{d}, J=3.0 \mathrm{~Hz}, \mathrm{H}-3) ;{ }^{13} \mathrm{C}$ NMR ( $\left.\mathrm{CDCl}_{3}, 100 \mathrm{MHz}\right) \delta 55.80$ (C-4a), 56.33 (C-1a), 64.05 (C-9), 66.65 (C-10), 82.77 (C-7), 90.83 (C-8), 111.57 (C-2), 11.87 (C-6), 116.23 (C-5), 118.09 (C-3), 153.16 (C-1), 154.62 (C-4); HRESIMS $m / z 205.0871\left[\mathrm{M}-\mathrm{H}_{2} \mathrm{O}\right]^{+}$(calcd for $\mathrm{C}_{12} \mathrm{H}_{14} \mathrm{O}_{4} 205.0892$ ).

General Procedure for the Grignard Reaction. ${ }^{23}$ Compound 9 (150 mg, $0.599 \mathrm{mmol})$ or $18(50 \mathrm{mg}, 0.26 \mathrm{mmol})$ in dry THF $(7.5 \mathrm{~mL})$ was placed in a flame-dried round-bottomed flask under an argon atmosphere at $0{ }^{\circ} \mathrm{C}$. The mixture was stirred for $5 \mathrm{~min}$, and $\mathrm{MeMgBr}$ in THF ( $1 \mathrm{M}, 1.5$ equiv) was added dropwise to the solution over $5 \mathrm{~min}$. The reaction mixture was warmed to rt and stirred for $2.5 \mathrm{~h}$. The mixture was cooled to $0{ }^{\circ} \mathrm{C}$, quenched with saturated $\mathrm{NH}_{4} \mathrm{Cl}$, and stirred for a further $30 \mathrm{~min}$. The aqueous layer was extracted with $\mathrm{EtOAc}(3 \times 15 \mathrm{~mL})$. The combined organic layers were washed with brine and dried over anhydrous $\mathrm{Na}_{2} \mathrm{SO}_{4}$. Evaporation of the solvent gave a residue, which was purified on Sephadex LH-20 eluting with $95 \% \mathrm{CH}_{2} \mathrm{Cl}_{2} /$ hexanes to give compound 15 ( $159 \mathrm{mg}, 99 \%$ yield) or compound 19 ( $54 \mathrm{mg}, 99 \%$ yield) as a yellow oil.

4-[2,5-Bis(methoxymethoxy)phenyl]but-3-yn-2-ol ${ }^{23}$ (15). IR $(\mathrm{KBr}) \nu_{\max } 3398,2226 \mathrm{~cm}^{-1}$; ${ }^{1} \mathrm{H} \mathrm{NMR}\left(\mathrm{CDCl}_{3}, 400 \mathrm{MHz}\right) \delta 1.51$ $(3 \mathrm{H}, \mathrm{d}, J=6.6 \mathrm{~Hz}, \mathrm{H}-10), 3.43$ (3H, s, H- $4 \mathrm{~b}), 3.48$ (3H, s, H-1b), 4.74
$(1 \mathrm{H}, \mathrm{q}, J=6.6 \mathrm{~Hz}, \mathrm{H}-9), 5.06$ (3H, s, H-4a), 5.14 (3H, s, H-1a), 6.90 $(1 \mathrm{H}, \mathrm{dd}, J=9.0$ and $3.0 \mathrm{~Hz}, \mathrm{H}-5), 6.98(1 \mathrm{H}, \mathrm{d}, J=9.0 \mathrm{~Hz}, \mathrm{H}-6), 7.06$ $(1 \mathrm{H}, \mathrm{d}, J=3.0 \mathrm{~Hz}, \mathrm{H}-3) ;{ }^{13} \mathrm{C} \mathrm{NMR}\left(\mathrm{CDCl}_{3}, 100 \mathrm{MHz}\right) \delta 24.28(\mathrm{C}-10)$, 55.91 (C-4b), 56.25 (C-1b), 58.70 (C-9), 79.86 (C-7), 94.96 (C-4a), 95.41 (C-8), 95.83 (C-1a), 114.33 (C-2), 117.25 (C-6), 118.23 (C-5), 120.94 (C-3), 151.87 (C-4), 152.81 (C-1); HRESIMS $m / z 289.1057$ $[\mathrm{M}+\mathrm{Na}]^{+}$(calcd for $\left.\mathrm{C}_{14} \mathrm{H}_{18} \mathrm{O}_{5} 289.1154\right)$.

4-(2,5-Dimethoxyphenyl)but-3-yn-2-ol ${ }^{23}$ (19). IR (KBr) $\nu_{\max } 3427$, $2193 \mathrm{~cm}^{-1} ;{ }^{1} \mathrm{H} \mathrm{NMR}\left(\mathrm{CDCl}_{3}, 400 \mathrm{MHz}\right) \delta 1.57(3 \mathrm{H}, \mathrm{d}, J=7.0 \mathrm{~Hz}, \mathrm{H}-$ 10), $3.76(3 \mathrm{H}, \mathrm{s}, \mathrm{H}-4 \mathrm{a}), 3.83(3 \mathrm{H}, \mathrm{s}, \mathrm{H}-1 \mathrm{a}), 4.80(1 \mathrm{H}, \mathrm{q}, J=6.6 \mathrm{~Hz}, \mathrm{H}-9)$, $6.79(1 \mathrm{H}, \mathrm{d}, J=9.0 \mathrm{~Hz}, \mathrm{H}-6), 6.84(1 \mathrm{H}, \mathrm{dd}, J=9.0$ and $3.0 \mathrm{~Hz}, \mathrm{H}-5), 6.94$ $(1 \mathrm{H}, \mathrm{d}, J=3.0 \mathrm{~Hz}, \mathrm{H}-3) ;{ }^{13} \mathrm{C}$ NMR $\left(\mathrm{CDCl}_{3}, 100 \mathrm{MHz}\right) \delta 24.46(\mathrm{C}-10)$, 55.93 (C-4a), 56.55 (C-1a), 59.14 (C-9), 80.35 (C-7), 95.22 (C-8), 112.16 (C-6), 112.35 (C-2), 116.0 (C-5), 118.46 (C-3), 153.31 (C-1), 154.42 (C-4); HRESIMS $m / z 189.0918\left[\mathrm{M}-\mathrm{H}_{2} \mathrm{O}+\mathrm{H}\right]^{+}$(calcd for $\mathrm{C}_{12} \mathrm{H}_{14} \mathrm{O}_{3}$ 189.0943).

Dehydration Methodology. ${ }^{24}$ (Carboxysulfamoyl)triethylammonium hydroxide inner salt methyl ester ${ }^{34,35}$ (Burgess' reagent) $(70 \mathrm{mg}, 0.29 \mathrm{mmol}, 3.9$ equiv) in dry benzene $(2 \mathrm{~mL})$ and $15(20 \mathrm{mg}$, $0.19 \mathrm{mmol})$ in dry benzene $(1 \mathrm{~mL})$ were placed in a flame-dried roundbottomed flask under an argon atmosphere. The mixture was heated under reflux at $110^{\circ} \mathrm{C}$ for $18 \mathrm{~h}$. A further portion of Burgess' reagent ( $54 \mathrm{mg}, 0.23 \mathrm{mmol}, 3$ equiv) was added to the reaction mixture, and this was heated under reflux for $24 \mathrm{~h}$. Finally, an additional 3 equiv of Burgess' reagent was added to the reaction mixture, and this was heated under reflux for $24 \mathrm{~h}$. The reaction mixture was cooled to rt, quenched with saturated brine $(5 \mathrm{~mL})$, and stirred for a further $30 \mathrm{~min}$, and the organic layer was decanted off. The aqueous layer was extracted with EtOAc $(3 \times 20 \mathrm{~mL})$, and the combined organic layers were washed with brine, dried over anhydrous $\mathrm{MgSO}_{4}$, and evaporated under reduced pressure. The crude material was purified by silica gel column chromatography, eluting with $15 \%$ EtOAc/hexanes, to give compound $14(15.1 \mathrm{mg}, 81 \%$ yield $)$ as a yellow oil.

2-But-3-en-1-yn-1-yl-1,4-bis(methoxymethoxy)benzene (14). IR $(\mathrm{KBr}) \nu_{\max } 2330,1741 \mathrm{~cm}^{-1} ;{ }^{1} \mathrm{H}$ NMR $\left(\mathrm{CDCl}_{3}, 400 \mathrm{MHz}\right) \delta 3.46$ $(3 \mathrm{H}, \mathrm{s}, \mathrm{H}-4 \mathrm{~b}), 3.52$ (3H, s, H-1b), 5.10 (3H, s, H-4a), 5.17 (3H, s, H-1a), $5.53(1 \mathrm{H}, \mathrm{dd}, J=11.1$ and $2.1 \mathrm{~Hz}, \mathrm{H}-10), 5.73(1 \mathrm{H}, \mathrm{dd}, J=17.5$ and $2.1 \mathrm{~Hz}, \mathrm{H}-10), 6.04(1 \mathrm{H}, \mathrm{dd}, J=17.5$ and $11.1 \mathrm{~Hz}, \mathrm{H}-9), 6.93(1 \mathrm{H}, \mathrm{dd}$, $J=9.0$ and $3.0 \mathrm{~Hz}, \mathrm{H}-5), 7.02(1 \mathrm{H}, \mathrm{d}, J=9.0 \mathrm{~Hz}, \mathrm{H}-6), 7.11(1 \mathrm{H}, \mathrm{d}, J=$ $3.0 \mathrm{~Hz}, \mathrm{H}-3) ;{ }^{13} \mathrm{C} \mathrm{NMR}\left(\mathrm{CDCl}_{3}, 100 \mathrm{MHz}\right) \delta 56.08(\mathrm{C}-4 \mathrm{~b}), 56.39(\mathrm{C}-$ 1b), 86.21 (C-8), 92.17 (C-7), 95.17 (C-4a), 96.03 (C-1a), 114.97 (C2), 117.40 (C-6), 117.47 (C-9), 118.40 (C-5), 120.96 (C-3), 127.06 (C10), 152.05 (C-4), 152.95 (C-1); HRESIMS $m / z 287.0905[\mathrm{M}+\mathrm{Na}]^{+}$ (calcd for $\mathrm{C}_{14} \mathrm{H}_{16} \mathrm{O}_{5} 287.0896$ ).

2-But-3-en-1-yn-1-yl-1,4-dimethoxybenzene (21). Yield 80\%; colorless oil; IR $(\mathrm{KBr}) \nu_{\max } 2194 \mathrm{~cm}^{-1}$; ${ }^{1} \mathrm{H} \mathrm{NMR}\left(\mathrm{CDCl}_{3}, 500 \mathrm{MHz}\right)$ $\delta 3.75(1 \mathrm{H}, \mathrm{s}, \mathrm{H}-4 \mathrm{a}), 3.84(1 \mathrm{H}, \mathrm{s}, \mathrm{H}-1 \mathrm{a}), 5.53(1 \mathrm{H}, \mathrm{dd}, J=11.2$ and 2.1 $\mathrm{Hz}, \mathrm{H}-10), 5.75(1 \mathrm{H}, \mathrm{dd}, J=17.5$ and $2.1 \mathrm{~Hz}, \mathrm{H}-10), 6.06(1 \mathrm{H}, \mathrm{dd}, J=$ 17.5 and $11.2 \mathrm{~Hz}, \mathrm{H}-9), 6.79(1 \mathrm{H}, \mathrm{d}, J=9.0 \mathrm{~Hz}, \mathrm{H}-6), 6.83(1 \mathrm{H}, \mathrm{dd}, J=$ 9.0 and $2.9 \mathrm{~Hz}, \mathrm{H}-5), 6.95(1 \mathrm{H}, \mathrm{d}, J=2.9 \mathrm{~Hz}, \mathrm{H}-3) ;{ }^{13} \mathrm{C} \mathrm{NMR}\left(\mathrm{CDCl}_{3}\right.$, $125 \mathrm{MHz}) \delta 56.43$ (C-1a), 55.79 (C-4a), 86.17 (C-7), 92.09 (C-8), 111.97 (C-2), 112.75 (C-9), 115.84 (C-6), 117.33 (C-5), 118.1 (C-3), 126.84 (C-10), 153.18 (C-4), 154.38 (C-1); HRESIMS $m / z 189.0920$ $[\mathrm{M}+\mathrm{H}]^{+}$(calcd for $\mathrm{C}_{12} \mathrm{H}_{12} \mathrm{O}_{2}$ 189.0916).

Deprotection Study. 4-[2,5-Bis(methoxymethoxy)phenyl]-2-methoxy-2-methylbut-3-yn-1-ol (12). Compound 10 (0.1 mmol) in $\mathrm{MeOH}(2.8 \mathrm{~mL})$ was placed in a flame-dried round-bottomed flask under an argon atmosphere. $\mathrm{H}_{2} \mathrm{SO}_{4}(95 \mu \mathrm{L})$ was added dropwise to the solution, and this was stirred for $30 \mathrm{~min}$. The reaction mixture was quenched with saturated $\mathrm{NH}_{4} \mathrm{Cl}$ and stirred for a further $30 \mathrm{~min}$, and the organic layer was separated. The aqueous layer was extracted with EtOAc $(3 \times 10 \mathrm{~mL})$. The combined organic layers were washed with brine, dried over anhydrous $\mathrm{Na}_{2} \mathrm{SO}_{4}$, and evaporated under reduced pressure. The crude material was purified by silica gel column chromatography, eluting with $60 \% \mathrm{EtOAc} /$ hexanes, to give compound $12(27.9 \mathrm{mg}, 90 \%$ yield $)$ as a colorless oil, which was highly air-sensitive (the compound needs to be frozen and kept in the dark under argon). IR $(\mathrm{KBr}) \nu_{\max } 3336,2197 \mathrm{~cm}^{-1}{ }^{1}{ }^{1} \mathrm{H} \mathrm{NMR}\left(\mathrm{CD}_{3} \mathrm{OD}, 400 \mathrm{MHz}\right) \delta 3.43(3 \mathrm{H}$, s, H-11), $3.70(2 \mathrm{H}, \mathrm{m}, \mathrm{H}-10), 4.26(1 \mathrm{H}, \mathrm{dd}, J=6.3$ and $5.1 \mathrm{~Hz}, \mathrm{H}-9)$, $6.73(1 \mathrm{H}, \mathrm{s}, \mathrm{H}-3), 6.73(1 \mathrm{H}, \mathrm{d}, J=1.0 \mathrm{~Hz}, \mathrm{H}-5), 6.78(1 \mathrm{H}, \mathrm{dd}, J=2.4$ and 
$1.1 \mathrm{~Hz}, \mathrm{H}-6) ;{ }^{13} \mathrm{C}$ NMR $\left(\mathrm{CD}_{3} \mathrm{OD}, 100 \mathrm{MHz}\right) \delta 55.75$ (C-11), 64.83 (C10), 73.28 (C-9), 82.53 (C-7), 90.46 (C-8), 109.74 (C-2), 116.16 (C-6), 117.50 (C-5), 117.97 (C-3), 149.95 (C-4), 151.53 (C-1); HRESIMS $m / z$ 207.0714 $[\mathrm{M}-\mathrm{H}]^{+}$(calcd for $\mathrm{C}_{11} \mathrm{H}_{12} \mathrm{O}_{4}$ 207.0736).

Speciosin P. Compound 11 (22 mg, $0.078 \mathrm{mmol})$ in $\mathrm{MeOH}(1 \mathrm{~mL})$ was placed in a flame-dried round-bottomed flask under an argon atmosphere, and $\mathrm{HCl}_{[33 \%]}(10 \mu \mathrm{L})$ was added to the solution. The reaction mixture was heated under reflux at $70{ }^{\circ} \mathrm{C}$ for $2 \mathrm{~h}$. The mixture was cooled to rt, and saturated $\mathrm{KHCO}_{3}(5 \mathrm{~mL})$ was added to adjust the $\mathrm{pH}$ of the solution to 7. The aqueous phase was extracted with EtOAc $(4 \times 10 \mathrm{~mL})$. The combined organic layers were washed with brine, dried over anhydrous $\mathrm{Na}_{2} \mathrm{SO}_{4}$, and evaporated under reduced pressure. The crude material was purified by silica gel column chromatography, eluting with $40 \% \mathrm{CHCl}_{3}$ /acetone, to give the natural product $( \pm$-speciosin P (2) (15.1 mg, 90\% yield) as a colorless oil. The spectroscopic data corresponded to the literature data. ${ }^{2}$

2-(3-Hydroxyprop-1-yn-1-yl)benzene-1,4-diol (23). Compound 8 $(20 \mathrm{mg}, 0.079 \mathrm{mmol})$ in $\mathrm{MeOH}(1 \mathrm{~mL})$ was placed in a flame-dried round-bottomed flask under an argon atmosphere, and $\mathrm{HCl}_{[33 \%]}$ $(10 \mu \mathrm{L})$ was added to the solution. The reaction mixture was heated under reflux at $70^{\circ} \mathrm{C}$ for $2 \mathrm{~h}$ under argon. The mixture was cooled to rt, and saturated $\mathrm{KHCO}_{3}(5 \mathrm{~mL})$ was added to adjust the $\mathrm{pH}$ of the solution to 7. The aqueous phase was extracted with EtOAc $(4 \times 10$ $\mathrm{mL}$ ), and the combined organic layers were washed with brine, dried over anhydrous $\mathrm{Na}_{2} \mathrm{SO}_{4}$, and evaporated under reduced pressure. The crude material was purified by silica gel column chromatography, eluting with $40 \% \mathrm{CHCl}_{3}$ /acetone, to give $\mathbf{2 3}$ (12 $\mathrm{mg}$, 94\% yield) as a colorless oil: IR (KBr) $\nu_{\max } 3270,2193 \mathrm{~cm}^{-1}$; ${ }^{1} \mathrm{H}$ NMR $\left(\mathrm{CD}_{3} \mathrm{OD}, 400 \mathrm{MHz}\right) \delta$ $4.41(2 \mathrm{H}, \mathrm{s}, 9-\mathrm{H}), 6.63(1 \mathrm{H}, \mathrm{dd}, J=8.0$ and $2.6 \mathrm{~Hz}, 3-\mathrm{H}), 6.67(1 \mathrm{H}, \mathrm{d}, J=$ $8.0 \mathrm{~Hz}, 5-\mathrm{H}), 6.71(1 \mathrm{H}, \mathrm{dd}, J=2.6 \mathrm{~Hz}, 3-\mathrm{H}) ;{ }^{13} \mathrm{C}$ NMR $\left(\mathrm{CD}_{3} \mathrm{OD}, 100\right.$ $\mathrm{MHz}) \delta 51.43$ (C-9), 81.92 (C-7), 92.58 (C-8), 111.53 (C-2), 117.15 (C-6), 118.22 (C-3), 119.40 (C-5), 151.03 (C-4), 152.47 (C-1); HRESIMS $m / z 163.0387[\mathrm{M}-\mathrm{H}]^{+}\left(\right.$calcd for $\left.\mathrm{C}_{9} \mathrm{H}_{8} \mathrm{O}_{3} 163.0395\right)$.

2-(3-Hydroxybut-1-yn-1-yl)benzene-1,4-diol (24). Yield 90\%; colorless oil; IR $(\mathrm{KBr}) \nu_{\max } 3342,2197 \mathrm{~cm}^{-1}$; ${ }^{1} \mathrm{H}$ NMR $\left(\mathrm{C}_{2} \mathrm{D}_{6} \mathrm{CO}\right.$, $500 \mathrm{MHz}) \delta 1.44(3 \mathrm{H}, \mathrm{d}, J=6.6 \mathrm{~Hz}, \mathrm{H}-10), 4.69(1 \mathrm{H}, \mathrm{q}, J=6.6 \mathrm{~Hz}, \mathrm{H}-9)$, $6.70(1 \mathrm{H}, \mathrm{dd}, J=8.7$ and $2.7 \mathrm{~Hz}, \mathrm{H}-5), 6.73(1 \mathrm{H}, \mathrm{dd}, J=8.7$ and $0.8 \mathrm{~Hz}, \mathrm{H}-$ 5), $6.75(1 \mathrm{H}, \mathrm{dd}, J=2.7$ and $0.8 \mathrm{~Hz}, \mathrm{H}-3) ;{ }^{13} \mathrm{C} \mathrm{NMR}\left(\mathrm{C}_{2} \mathrm{D}_{6} \mathrm{CO}, 125 \mathrm{MHz}\right)$ $\delta 24.94$ (C-10), 58.64 (C-9), 79.33 (C-7), 97.73 (C-8), 111.10 (C-2), 116.99 (C-6), 118.05 (C-5), 118.78 (C-3), 150.89 (C-4), 152.06 (C-1); HRESIMS $m / z 179.0713[\mathrm{M}+\mathrm{H}]^{+}\left(\right.$calcd for $\left.\mathrm{C}_{10} \mathrm{H}_{10} \mathrm{O}_{3} 179.0709\right)$.

Speciosin G. Compound $14(20 \mathrm{mg}, 0.081 \mathrm{mmol})$ in $\mathrm{MeOH}(2 \mathrm{~mL})$ was placed in a flame-dried round-bottomed flask under an argon atmosphere, $\mathrm{HCl}_{[33 \%]}(20 \mu \mathrm{L})$ was added to the solution, and the reaction mixture was heated under reflux at $70{ }^{\circ} \mathrm{C}$ for $90 \mathrm{~min}$. The mixture was cooled to rt, and saturated aqueous $\mathrm{KHCO}_{3}(5 \mathrm{~mL})$ was added to adjust the $\mathrm{pH}$ of the solution to 7 . The aqueous phase was extracted with EtOAc $(4 \times 10 \mathrm{~mL})$. The combined organic layers were washed with brine, dried over anhydrous $\mathrm{Na}_{2} \mathrm{SO}_{4}$, and evaporated under reduced pressure. The crude material was purified by HPLC D7000, eluting with $30 \% \mathrm{EtOAc} /$ hexanes, to give the natural product speciosin $\mathrm{G}$ (1) (11.6 $\mathrm{mg}, 90 \%$ yield) (retention time $12.3 \mathrm{~min}$ ) as a colorless oil. The spectroscopic data corresponded to the literature data. ${ }^{1}$

Wheat Coleoptile Bioassays. Wheat seeds (Triticum aestivum L. cv. Catervo) were sown in $15 \mathrm{~cm}$ diameter Petri dishes moistened with $\mathrm{H}_{2} \mathrm{O}$ and grown in the dark at $22 \pm 1{ }^{\circ} \mathrm{C}$ for 3 days. ${ }^{36}$ The roots and caryopses were removed from the shoots and were placed in a Van der Weij guillotine, and the apical $2 \mathrm{~mm}$ was cut off and discarded. The next $4 \mathrm{~mm}$ of the coleoptiles was removed and used for the bioassay. All manipulations were performed under a green safelight. ${ }^{37}$ Test solutions of each compound were prepared in a phosphate-citrate buffer solution containing $2 \%$ sucrose adjusted to $\mathrm{pH} 5.6$ at concentrations of 1000 , $333,100,33$, and $10 \mu \mathrm{M}$. Parallel controls were also run. Five coleoptiles were placed in each test tube containing test or control solutions $(2 \mathrm{~mL})$ (three replicates per dilution). The test tubes were rotated at $0.25 \mathrm{rpm}$ on a roller tube apparatus for $24 \mathrm{~h}$ at $22^{\circ} \mathrm{C}$ in the dark. The coleoptiles were digitally photographed and measured. Data were statistically analyzed using Welch's test, ${ }^{38}$ and results are presented as percentage differences from control. Thus, zero represents the control, positive values represent growth stimulation, and negative values represent growth inhibition.

\section{ASSOCIATED CONTENT}

\section{Supporting Information}

Analytical data including ${ }^{1} \mathrm{H}$ and ${ }^{13} \mathrm{C}$ NMR spectra and selected mass spectra for new compounds are illustrated. This material is available free of charge via the Internet at http://pubs.acs.org.

\section{AUTHOR INFORMATION}

\section{Corresponding Author}

*Tel: +34-956012770. Fax: +34-956016288. E-mail: famacias@ uca.es.

\section{Notes}

The authors declare no competing financial interest.

\section{ACKNOWLEDGMENTS}

The authors gratefully acknowledge the Spanish Ministry of Science and Technology, Project No. AGL2009-08864/AGR, and Consejería de Innovación, Ciencia e Industria (P07-FQM-03031). We also acknowledge the Spanish Ministry of Science and Technology for fellowships.

\section{REFERENCES}

(1) Jiang, M. Y.; Zhang, L.; Liu, R.; Dong, Z. J.; Liu, J. K. J. Nat. Prod. 2009, 72, 1405-1409.

(2) Jiang, M.-Y.; Li, Y.; Wang, F.; Liu, J.-K. Phytochemistry 2011, 72, 923-928.

(3) Ishibashi, K.; Nose, K.; Shindo, T.; Arai, M.; Mishima, H. Sankyo Kenkyusho Nempo 1968, 20, 76-79.

(4) Kupka, J.; Anke, T.; Steglich, W.; Zechlin, L. J. Antibiot. 1981, 34, 298-304.

(5) Kim, J. H.; Mahoney, N.; Chan, K. L.; Molyneux, R. J.; Campbell, B. C. Curr. Microbiol. 2004, 49, 282-287.

(6) Liu, S.; Guo, L.; Che, Y.; Liu, L. Fitoterapia 2013, 85, 114-118.

(7) Pulley, S. R.; Czako, B. Tetrahedron Lett. 2004, 45, 5511-5514.

(8) Pinault, M.; Frangin, Y.; Genet, J. P.; Zamarlik, H. Synthesis (Stuttgart, Ger.) 1990, 935-937.

(9) Viciu, M. S.; Nolan, S. P. In Modern Arylation Methods; Ackermann, L., Ed.; Wiley-VCH: Weinheim, Germany, 2009; Chapter 6, pp 183220.

(10) Chinchilla, R.; Najera, C. Chem. Rev. 2007, 107, 874-922.

(11) Chinchilla, R.; Najera, C. Chem. Soc. Rev. 2011, 40, 5084-5121.

(12) Feldman, K. S.; Selfridge, B. R. Heterocycles 2010, 81, 117-143.

(13) Shet, J.; Desai, V.; Tilve, S. Synthesis 2004, 11, 1859-1863.

(14) Corey, E. J.; Suggs, J. W. Tetrahedron Lett. 1975, 16, 2647-2650.

(15) Macías, F. A.; Carrera, C.; Chinchilla, N.; Fronczek, F. R.; Galindo, J. C. G. Tetrahedron 2010, 66, 4125-4132.

(16) Dess, D. B.; Martin, J. C. J. Org. Chem. 1983, 48, 4155-4156.

(17) Mancuso, A. J.; Huang, S.-L.; Swern, D. J. Org. Chem. 1978, 43, 2480-2482.

(18) Corey, E. J.; Chaykovsky, M. J. Am. Chem. Soc. 1965, 87, 13531364.

(19) Corey, E. J.; Chaykovsky, M. J. Am. Chem. Soc. 1962, 84, 37823783.

(20) Guerrero-Vásquez, G. A.; Andrade, C. K. Z.; Molinillo, J. M. G.; Macías, F. A. Eur. J. Org. Chem. 2013, 2013, 6175-6180.

(21) Pilichowski, J.-F.; Morel, M.; Tamboura, F.; Chmela, S.; Baba, M.; Lacoste, J. Polym. Degrad. Stab. 2010, 95, 1575-1580.

(22) Hrobarikova, V.; Hrobarik, P.; Gajdos, P.; Fitilis, I.; Fakis, M.; Persephonis, P.; Zahradnik, P. J. Org. Chem. 2010, 75, 3053-3068.

(23) Naito, J.; Yamamoto, Y.; Akagi, M.; Sekiguchi, S.; Watanabe, M.; Harada, N. Monatsh. Chem. 2005, 136, 411-445.

(24) Leverett, C. A.; Purohit, V. C.; Johnson, A. G.; Davis, R. L.; Tantillo, D. J.; Romo, D. J. Am. Chem. Soc. 2012, 134, 13348-13356.

(25) Nebo, L.; Varela, R. M.; Molinillo, J. M. G.; Sampaio, O. M.; Severino, V. G. P.; Cazal, C. M.; Fernandes, M. F. d. G.; Fernandes, J. B.; Macías, F. A. Phytochem. Lett. 2014, 8, 226-232. 
(26) Tang, X.; Woodward, S.; Krause, N. Eur. J. Org. Chem. 2009, 2009, $2836-2844$.

(27) Smith, L. R.; Mahoney, N.; Molyneux, R. J. J. Nat. Prod. 2003, 66, 169-176.

(28) Cutler, H. G. Proc. Plant Growth Regul. Soc. Am. 11th 1984, 1-9.

(29) Cutler, S. J.; Hoagland, R. E.; Cutler, H. G. In Evaluation of Selected Pharmaceuticals as Potential Herbicides: Bridging the Gap between Agrochemicals and Pharmaceuticals; Kluwer Academic Publishers: 2000; pp 129-137.

(30) Jacyno, J. M.; Cutler, H. G. PGRSA Q. 1993, 21, 15-24.

(31) Pulley, S. R.; Czako, B. Tetrahedron Lett. 2004, 45, 5511-5514.

(32) Franks, M. A.; Schrader, E. A.; Pietsch, E. C.; Pennella, D. R.; Torti, S. V.; Welker, M. E. Bioorg. Med. Chem. 2005, 13, 2221-2233.

(33) Fleming, I.; Rowley, M.; Cuadrado, P.; González-Nogal, A. M.; Pulido, F. J. Tetrahedron 1989, 45, 413-424.

(34) Raghavan, S.; Mustafa, S.; Rathore, K. Tetrahedron Lett. 2008, 49, $4256-4259$.

(35) Burgess, E. M.; Penton, H. R.; Taylor, E. A. J. Org. Chem. 1973, 38, 26-31.

(36) Hancock, C. R.; Barlow, H. W. B.; Lacey, H. J. J. Exp. Bot. 1964, $15,166-176$.

(37) Nitsch, J. P.; Nitsch, C. Plant Physiol. 1956, 31, 94-111.

(38) Martín Andrés, A.; Luna del Castillo, J. D. Bioestadistica para las Ciencias de la Salud; Norma: Madrid, 1990. 